\title{
A Pull Based Energy Efficient Data Aggregation Scheme for Wireless Sensor Networks
}

\author{
C. Kavitha \\ Assistant Professor, R.V.College of \\ Engineering \\ Visvesvaraya Technological University, \\ Karnataka, India
}

\author{
Dr.K.V.Viswanatha \\ Professor, Department of CSE, \\ R.V.College of Engineering \\ Visvesvaraya Technological University, \\ Karnataka, India
}

\begin{abstract}
In case of wireless routing in sensor networks, data aggregation has been proposed as a predominantly constructive prototype. Most of the routing algorithms for traditional networks are address centric, and the ad hoc nature of wireless sensor network makes them unsuitable for practical applications. Data-centric technologies that carry out in-network aggregation of data to capitulate energyefficient dissemination are essential. In this paper, we propose a Pull based Energy Efficient Data Aggregation (PEEDA) approach, to effectively deliver the data to the sink. In this approach, the sink will broadcast an interest message containing its required data model, to all the nodes. We form an cost effective aggregation tree towards the sink based on the ToD structure. When the aggregator receives the data from the sources, it aggregates the data depending on the interest message using spatial and temporal convergence. To achieve energy efficient aggregation, the MAC protocol uses the partially overlapped channels. By simulation results, we show that the proposed scheme consumes less energy and reduces the overhead and delay.
\end{abstract}

The full text of the article is not available in the cache. Kindly refer the IJCA digital library at www.ijcaonline.org for the complete article. In case, you face problems while downloading the full-text, please send a mail to editor at editor@ijcaonline.org 